\title{
Research on the Key Technologies of Mobile Learning System
}

\author{
Cui Mei Li ${ }^{1, a}$, Le Huang ${ }^{2, b}$ and Rou Wang ${ }^{3, c}$ \\ ${ }^{1}$ School of Communication and Electronic, Jiangxi Science \& Technology Normal University \\ Nanchang, 330013, China \\ ${ }^{2}$ Grade 12 of School of Computer Science, Central China Normal University, Wuhan 430079, China \\ ${ }^{3}$ Department of Humanities, Nanchang Teachers College, Nanchang 330103, China \\ a996506207@qq.com, bbaidulele@163.com, ${ }^{\mathrm{c}} 171027349 @ q q . c o m$
}

Keywords: Mobile learning; Mobile web; WAP; Mobile streaming media; Mobile video communication

\begin{abstract}
Recently, with the rapid development of mobile computing technology, mobile learning is concerned by more and more people. Through mobile learning, learners could acquire the knowledge that they want at anytime and anywhere. In this paper, the key technologies of mobile learning system such as mobile Web technology, WAP technology, mobile streaming media technology and mobile video communication technology are expounded in detail. And thus provides a guidance of the theory, method and practice in the development and design of mobile learning systems.
\end{abstract}

\section{Introduction}

At the beginning of the 21st century, the mobile learning is concerned by more and more people and the novel emerging power brings an unprecedented learning experience. With the rapid popularization of mobile terminals, especially the android based platform, the mobile learning is provided the technical support [1].

Mobile learning breakthroughs the limitations of the time and distance, and makes a free learning style. Mobile learning is based on mobile computing devices such as mobile phone and other mobile terminals. Wireless communication technology and mobile terminal devices are used to provide education resources, education information and education services. Mobile learning meets the huge demand for episodic learning and makes the learning mode of 'Anywhere, Anytime, Anystyle and Anyone' (4A) reality [2, 3]. Mobile learning system is the foundation of the security of mobile learning's promotion and popularization. Mobile learning system is based on wireless communication technology, Internet technology and mobile computing technology, and provides the technology of software services for mobile education and teaching in all aspects [4, 5]. The key technologies of mobile learning, including mobile Web technology, WAP technology, mobile streaming media technology and mobile video communication technology, which have important implications for realizing all kinds of mobile learning system, would be stated in the following sections.

\section{Mobile Web technology}

The mobile web refers to accessing to the WWW's application, serves, and content at anytime and anywhere with the help of a mobile terminal equipment instead of a fixed connection. The mobile Internet based websites and the Wireless Markup Language (WML) written WAP websites are the examples of the mobile web. With the rapid development of mobile web technology, related web sites can be accessed through a mobile network related by the current most mobile devices. The widely used mobile website development technology mainly includes XHTML and WML, Full Web, etc. Developing mobile web applications rely on ASP.NET which provides a development framework and adaptive structure. Using the structure the mobile web browser outputs a particular code, and then presents the smaller part of the logical cutting. That makes the mobile web application better adapt the small screen on the mobile terminal devices. 


\section{WAP technology}

WAP is short for Wireless Application Protocol and which is an open global network communication protocol standards. The WAP is used to introduce the advance business and rich information on the Internet to the wireless terminal devices such as mobile phone and tablet PC, etc. The function of the WAP is to transform the Hyper Text Markup Language (HTML) described information on the Internet to the WML described information which can be displayed on the mobile terminal devices. The WAP only need the support of WAP proxy server and the mobile terminal devices, and the existing mobile communication protocol don't need to be changed, so it is widely used in many network types such as GSM, CDMA and TDMA.

The WAP experienced the WAP1.X and the WAP2.0 two versions, the latter is the trend. The WAP2.0 combined the latest technology and standards with the previous version, and applied in the wireless communication field. The technology and standards contains TCP/IP, HTTP/1.1, XHTML and TLS. The new services and applications such as the multimedia information services, data synchronization, unified storage interface, information configuration are added, meanwhile, the original functions such as Push technology, wireless technology application and user characteristics description are enhanced. The new features will bring different experience to people, and inspire the interest to the wireless communication. Fig. 1 shows the principle of the mobile phone access to the Internet through WAP1.X and the WAP2.0.

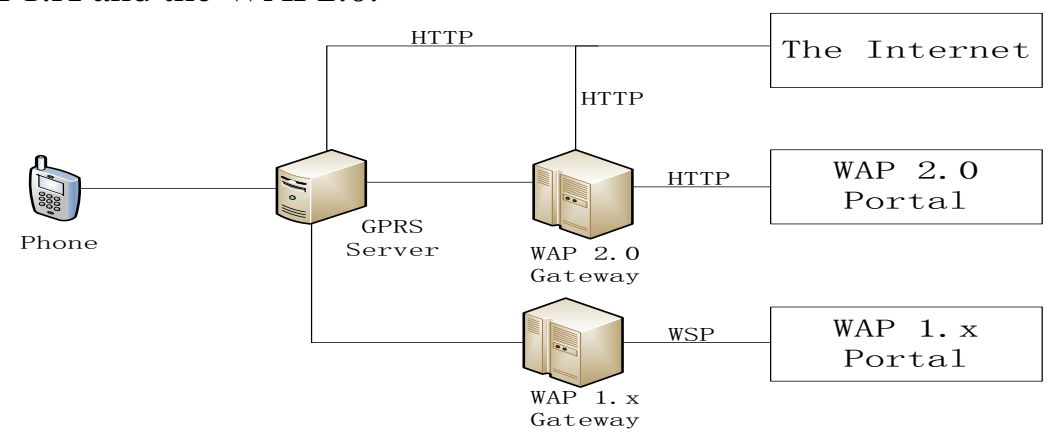

Fig. 1 The principle of the mobile phone access to the Internet through WAP1.X and the WAP2.0

\section{Mobile streaming media technology}

The mobile string media is to compress code the continuous audio and video multimedia data and storage the compressed data to the stream media server, then transmit the compress coding data to the mobile terminal devices according to various streaming media transmission protocols while ensuring that the streaming media transmission bandwidth matches with the mobile network bandwidth [6]. Before using streaming media technology, the entire file is not completely downloaded. But the downloaded part data content stored to the memory as the cached data. When the user plays the cached data, the mobile terminal downloads the remaining part of the data in the background from the stream media server. The stream media data is sent in real-time, i.e., the user can watch and download at the same time and don't need to wait until the multimedia files are completed download. Streaming media transmission protocol is the key technology of streaming media implementation.

There are two kinds of streaming media transmission, real-time streaming transmission and order streaming transmission, respectively. Order streaming download in a sequential way, that is, the user can download and watch the online program. However, the data only can be downloaded in order, so the user only can watch the download part and can't watch the other parts. In the real-time streaming transmission, the connection during transmission can be adjusted according to the user needs. The real-time stream transmission can ensure that the bandwidth of the network matched the bandwidth of the media signal. The real-time streaming transmission not only can transmit in real-time but also can randomly access, so it is very fit for live broadcast. Users are able to fast forward or backward, and periodically pause. 
The general mode of streaming transmission is to build connection with the Audio/Video (A/V) server through the two kinds of communication protocol, i.e., RTSP/TCP, RTP/UDP. Service will direct the output to the destination address of client where the $\mathrm{A} / \mathrm{V}$ player can be run. The streaming transmission system generally configures a set of dedicated server and player. The basic principle of streaming media transmission and the stream media transmission protocol are shown in Fig. 2 and Fig. 3 , respectively.

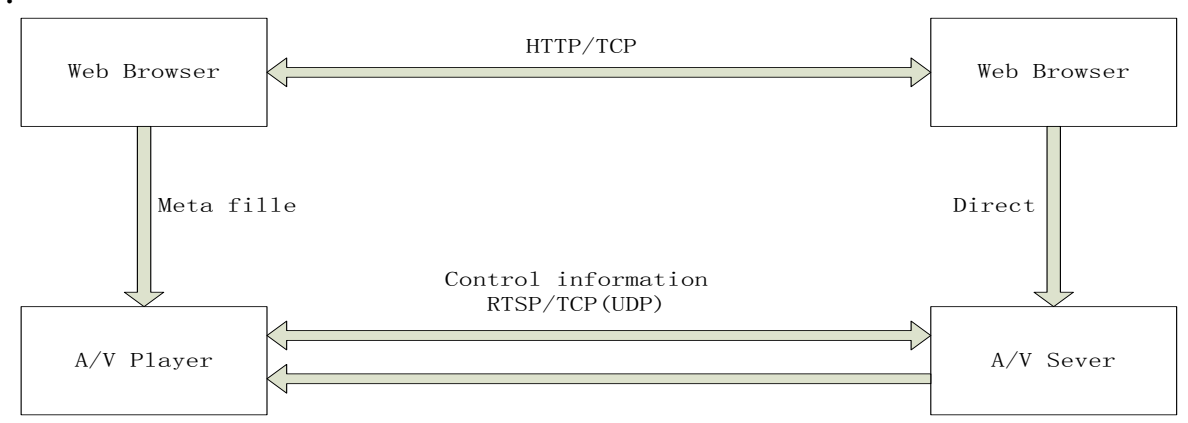

Fig. 2 The basic principle of streaming media transmission

\begin{tabular}{llll}
\hline \multirow{2}{*}{ RSVP } & RTP/RTCP & RTSP & SDP \\
\cline { 2 - 3 } & UDP & TCP
\end{tabular}

Fig. 3 The stream media transmission protocol

In the mobile communication network coverage area, the user's mobile terminal can freely access to multimedia data through streaming media and not restricted by the region. The streaming media formats are network version ASF/RM and mobile version 3GP/3GP2, etc. Among them, the mobile version of the $3 \mathrm{GP} / 3 \mathrm{GP} 2$ is supported by most terminal devices, and the ASF format is supported by less terminal devices. RM format is mainly used in low speed network to real-time transmit multimedia data, smoothly transmit and play multimedia data.

\section{Mobile video communication technology}

The popularity of the $3 \mathrm{G}$ technology and the mobile terminal devices with the function of video communication makes the face to face learning interaction possible [7, 8]. That leads to the learner can not only share study skills, exchange experience and answer question online, but also bring the important innovation on the way of study.

The mobile video communication is mainly composed of the mobile terminal devices which has the function of video communication and the mobile video communication server[9]. The mobile video communication terminal collects audio and video signals through a microphone and a camera respectively[10]. The video player is used to receive the video signal from the mobile video communication server. Every mobile terminal device has memory space and it would create a memory space as cache memory to cache the receiving audio, video data and the capturing audio and video data from microphone and camera. The mobile video communication server is mainly used for video and audio data's coding and storing. In order to ensure that the audio and video signals' synchronization and smooth in the mobile communication, the second level cache technology is usually used. After the mobile terminal devices receive the audio and video data, the data are extracted from the first level cache by split thread in the streaming media technology and then are segmented. The data package heads are added and form the data packages which are suit for mobile network transmission. Then the packages are transmitted based on the UDP protocol, and the response frame is been waited at the same time. On the other hand, after receive audio and video data, the received data are written in the second level cache area, and the response frame is been sent.

Except for sending audio and video data, the mobile video communication server needs to compression code the required send audio and video to the data streaming which are suiting for the mobile terminal devices. The process of compression coding is according to the registered mobile 
terminal devices' performance parameters. For example, the resolution and bit rate of the video data are adjusted to meet the various requirements of the different mobile terminal devices' performance parameters. Finally, data are transmitted to the targeted mobile terminal devices through the wireless communication network. The principle diagram of the mobile video communication is in Fig. 4.

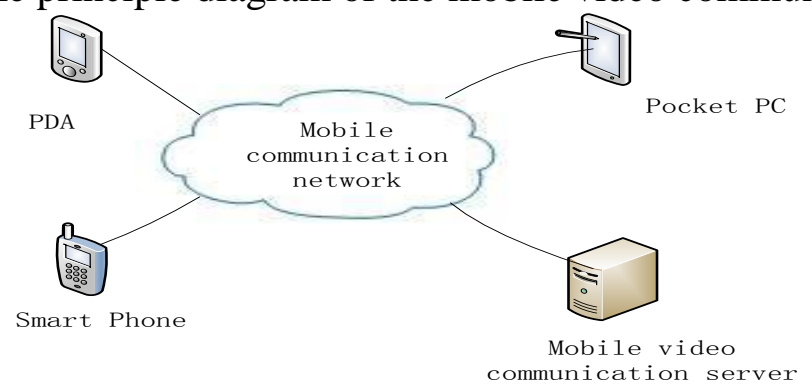

Fig. 4 The principle diagram of the mobile video communication

\section{Conclusion}

This work has detailed introduced the key technologies of mobile learning, i.e., mobile Web technology, WAP technology, mobile streaming media technology and mobile video communication technology. The mobile learning brings an unprecedented learning experience because it breakthroughs the limitations of the time and distance, and makes a free learning style. The mobile learning system satisfies the individualized learning needs of different learners and would facilitate building a lifelong learning society. Thanks to its advantages, mobile learning would be a learning trend in the future.

\section{Acknowledgements}

This work was supported by Key Project of the Educational Science Twelve-Five Year Plan of Jiangxi Province (Grant No.13ZD3L038), the Scientific and Technological Program of Jiangxi Provincial Education Development (Grant No. GJJ14583) and the Provincial Project of Teaching Reform and Research of Colleges and Universities in Jiangxi Province(Grant No. JXJG-13-10-15).

\section{References}

[1] Y. Jin. Research of one mobile learning system. Wireless Networks and Information Systems, 2009, International Conference on, IEEE, 2009: 162-165.

[2] M. Sharples, J. Taylor, G. Vavoula. Proceedings of mLearn 2005, 2005, 1(1): 1-9.

[3] Y. Park. The International Review of Research in Open and Distance Learning, 2011, 12(2): 78-102.

[4] W.H. Wu, J. Wu, C.Y. Chen, et al. Computers \& Education, 2012, 59(2): 817-827.

[5] N. M. Rao, C. Sasidhar, V.S. Kumar. arXiv preprint arXiv:1204.1594, 2012.

[6] G. Lawton. Computer, 2012, 45(2): 14-16.

[7] H.T. Dinh, C. Lee, D. Niyato, et al. Wireless communications and mobile computing, 2013, 13(18): 1587-1611.

[8] C.K. Looi, D. Sun, L. Wu. Computers \& Education, 2014, 77: 101-115.

[9] J. Park, H.J. Shim, B. Jeon, Broadband Multimedia Systems and Broadcasting (BMSB), 2011 IEEE International Symposium on , 2011: 8-10.

[10] J. Fredriksson, K. Groth, M. Rasanen, Computer-Based Medical Systems (CBMS), 2014 IEEE 27th International Symposium on ,2014:363-368. 